\title{
Effects of neoadjuvant chemotherapy on minimum alveolar concentration values of sevoflurane and desflurane in patients with hepatocellular carcinoma complicated with jaundice
}

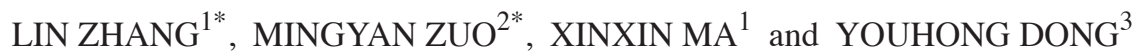 \\ Departments of ${ }^{1}$ Pharmacy, ${ }^{2}$ Pulmonary Disease and ${ }^{3}$ Oncology, Xiangyang No. 1 People's Hospital, \\ Hubei University of Medicine, Xiangyang, Hubei 441000, P.R. China
}

Received August 29, 2017; Accepted April 23, 2018

DOI: $10.3892 / \mathrm{ol} .2018 .8621$

\begin{abstract}
The effects of neoadjuvant chemotherapy on the minimum alveolar concentration (MAC) values of sevoflurane and desflurane in patients with hepatocellular carcinoma (HCC) complicated with jaundice were investigated. Eighty patients with HCC complicated with jaundice were selected. Forty patients underwent the neoadjuvant chemotherapy and were grouped into the desflurane group (Group D) and the sevoflurane group (Group S). Patients in all chemotherapy groups received 2 cycles of chemotherapy prior to surgery and underwent surgical treatment 3 weeks after chemotherapy. The remaining 40 patients in the control group were divided into the desflurane group (Group C1) and the sevoflurane group (Group C2). Changes in MAP, HR and BIS at different time points before and after anesthesia induction and skin incision were compared among the groups. Results showed that there were no significant differences in MAP, HR and BIS before anesthesia induction (T0) ( $\mathrm{P}>0.05)$; at each time point from $\mathrm{T} 1$ to T6, MAP, HR and BIS of Group D were significantly lower than those of Group $\mathrm{C} 1$ ( $\mathrm{P}>0.05)$. Furthermore, MAP, HR and BIS of Group $S$ were significantly lower than those of Group $\mathrm{C} 2$ ( $\mathrm{P}>0.05)$. The $\mathrm{MAC}_{\text {Mean }}$ of sevoflurane and desflurane were compared among all patient groups using the mean method. $\mathrm{MAC}_{\text {Mean }}$ values of Group D were significantly lower than those of Group $\mathrm{C} 1(\mathrm{P}<0.05)$. Notably, $\mathrm{MAC}_{\text {Dixon }}$ values of sevoflurane and desflurane were compared among all patient groups using the Dixon method and the differences were statistically significant $(\mathrm{P}<0.05)$. Logistic regression analyses were conducted, respectively, which revealed that the MAC of sevoflurane and
\end{abstract}

Correspondence to: Dr Xinxin Ma, Department of Pharmacy, Xiangyang No. 1 People's Hospital, Hubei University of Medicine, 15 Jiefang Road, Xiangyang, Hubei 441000, P.R. China

E-mail: maxinxin2016@163.com

*Contributed equally

Key words: anti-tumor combined chemotherapy regimen, anesthetic, inhalation, minimum alveolar effective concentration desflurane were associated with whether patients received the neoadjuvant chemotherapy. $\mathrm{MAC}_{\mathrm{Log}}$ of sevoflurane and desflurane were decreased in patients receiving the neoadjuvant chemotherapy. The results suggested that neoadjuvant chemotherapy can reduce MAC values of sevoflurane and desflurane in HCC patients complicated with jaundice and may improve these patients' sensitivity to sevoflurane and desflurane.

\section{Introduction}

Hepatic lesions of hepatocellular carcinoma (HCC) in the late stage can cause extensive hepatic failure and the compression of bile duct tumor, and jaundice occurs in about 10 to $40 \%$ of the patients. This change is often referred to as jaundice or cholestatic hepatocytes. Neoadjuvant chemotherapy not only narrows the scope of tumor surgeries, but also can significantly improve the survival rate of patients (1-3). Unlike other cancer patients, the central nervous system and peripheral nervous system of patients are damaged after neoadjuvant chemotherapy (4-6). At present, neoadjuvant chemotherapy has been clinically promoted, and one of the key issues of postoperative survival rate of patients is the depth of anesthesia, so exploring the impact of chemotherapy on patients is an important factor for safe anesthesia in patients. The target effect-site concentration $\mathrm{EC}_{50}$ of patients receiving neoadjuvant chemotherapy before operation is reduced compared with that of patients receiving no chemotherapy $(7,8)$. Sevoflurane and desflurane are relatively new types of inhaled anesthetics that have been widely used clinically $(9,10)$.

Desflurane has been widely used for the maintenance of general anesthesia for ambulatory surgery in adults and a type of inhalational agent with the least blood gas solubility coefficient and fastest recovery. However, desflurane has not been widely used in the pediatric population because of its two disadvantages: Its pungent smell and irritant nature, which makes it unsuitable for its use for induction of general anesthesia; and it association with inducing airway complications, such as a laryngospasm, breath holding, and cough (11). Because of the low blood-gas and blood-tissue solubility, sevoflurane has increasingly become more popular, and may provide rapid recovery after general anesthesia (12). Notably, sevoflurane is well known to cause emergence agitation (EA). 
A recent Cochrane review revealed that compared to sevoflurane, desflurane has a relative risk of EA of 1.46 with $95 \%$ confidence interval of 0.92-2.31 (13).

Due to changes in neuropathology caused by chemotherapy drugs, whether the neoadjuvant chemotherapy for patients will affect the minimum alveolar concentration (MAC) values of sevoflurane and desflurane during anesthesia needs to be further studied. Therefore, in this study, the effects of neoadjuvant chemotherapy drugs on sevoflurane and desflurane were investigated by the further study on the MAC values of sevoflurane and desflurane used to anesthetize HCC patients complicated with jaundice after neoadjuvant chemotherapy, to develop a much safer and more effective surgical program for HCC patients complicated with jaundice after neoadjuvant chemotherapy and provide a theoretical basis.

\section{Materials and methods}

General data. 80 HCC patients complicated with jaundice were selected, in which 40 patients received neoadjuvant chemotherapy. The present study was approved by the ethics committee of Xiangyang No. 1 People's Hospital, Hubei University of Medicine and informed consents were signed by the patients and/or guardians. The chemotherapy regimen was oxaliplatin combined with tegafur: $150 \mathrm{mg} / \mathrm{m}^{2}$ oxaliplatin was intravenously instilled for $3 \mathrm{~h}$ on the 1 st day; patients orally took tegafur after a meal for consecutive 14 days, and the initial dose was adjusted according to the body surface area of patients (the dose was adjusted to $40 \mathrm{mg} / \mathrm{m}^{2}$ for patients with less than $1.25 \mathrm{~m}^{2}$ body surface area; the dose was adjusted to $50 \mathrm{mg} / \mathrm{m}^{2}$ for patients with $1.25 \sim 1.5 \mathrm{~m}^{2}$ body surface area; the dose was adjusted to $60 \mathrm{mg} / \mathrm{m}^{2}$ for patients with more than $1.5 \mathrm{~m}^{2}$ body surface area). 21 days formed 1 cycle. The sevoflurane and desflurane treatment time for each patient was once. The concentration was from 0 to 2 times the concentration of the basis of MAC. The concentration was adjusted continuously by a special device.

Inclusion criteria: Patients whose American Society of Anesthesiologists (ASA) Score reached Grade I-II; HCC patients complicated with jaundice; patients with basically normal results in preoperative routine examinations; patients whose body mass index (BMI) was 22-23; patients aged 30-62 years old.

Exclusion criteria: Patients with a history of severe cardiovascular system or respiratory system disease, or renal dysfunction; patients with hepatic encephalopathy; patients with a history of mental or neurological disease; patients with a long history of taking psychiatric drugs or alcohol dependence; patients whose heart rate (HR) were less than $50 \mathrm{bpm}$ before skin incision; patients whose mean arterial blood pressure (MAP) was less than $50 \mathrm{mmHg}$; patients receiving drug intervention; patients whose intubation was not successfully conducted for the first time.

Grouping of subjects: A total of 40 patients receiving chemotherapy were randomly divided into the desflurane group (Group D, n=20) and the sevoflurane group (Group S, $\mathrm{n}=20$ ). Patients in all the chemotherapy groups were treated with chemotherapy for 2 cycles (14 days for 1 chemotherapy cycle), and received surgical treatment 3 weeks after chemotherapy. A total of 40 patients receiving no neoadjuvant chemotherapy were selected as the control group, and were randomly divided into the desflurane group (Group $\mathrm{C} 1, \mathrm{n}=20$ ) and the sevoflurane group (Group C2, n=20).

\section{Study methods}

Anesthesia methods. All patients did not undergo preoperative medication. Patients were infused with sodium chloride by injection via the peripheral venous route at a rate of $10 \mathrm{ml}$ $\mathrm{kg}^{-1} \mathrm{~h}^{-1}$. Electrocardiography, pulse, oxygen saturation and bispectral index (BIS) were monitored, and radial artery catheterization was performed under local anesthesia as an invasive method to measure arterial blood pressure. Anesthesia was induced by target-controlled infusion of propofol (Approval no.: National Medicine Permission no. H20123318; manufacturing enterprise: Xi'an Libang Pharmaceutical Co., Ltd.; target plasma concentration: $2 \mu \mathrm{g} / \mathrm{ml}$ ) and remifentanil [Approval no.: National Medicine Permission no. H20123422; manufacturing enterprise: China National Pharmaceutical Industrial Corporation Ltd., (Langfang Branch); target plasma concentration: $4 \mathrm{ng} / \mathrm{ml}$ ], and changes in consciousness of patients were carefully observed. Endotracheal intubation was carried out for mechanical ventilation. After that, patients began to inhale sevoflurane (Approval no.: National Medicine Permission no. H20070172; manufacturing enterprise: Shanghai Hengrui Pharmaceutical Co., Ltd.) and desflurane to maintain anesthesia, respectively, and the infusion of propofol and remifentanil was stopped. The volatilization pot was adjusted according to the end tidal concentrations of sevoflurane and desflurane so that the end tidal concentrations of them reached the target values and lasted for $15 \mathrm{~min}$ or longer. HR, MAP and BIS before anesthesia induction (T0), at 2 min before skin incision (T1), at $1 \mathrm{~min}$ before skin incision (T2), immediately before skin incision (T3), immediately after skin incision (T4), at $1 \mathrm{~min}$ after skin incision (T5) and at $2 \mathrm{~min}$ after skin incision were recorded, after which $0.15 \mathrm{mg} \mathrm{kg} \mathrm{kg}^{-1}$ cisatracurium (Approval no.: National Medicine Permission no. H20090202; manufacturing enterprise: Zhejiang Xianju Pharmaceutical Co., Ltd.) was used according to the operation condition.

Test methods. The mean values of HR, MAP and BIS measured at from T0 to T6 were calculated, and changes in HR, MAP and BIS at each time point were compared, which was recorded by Multifunctional monitor. If the amplitude of changes in the mean values of HR, MAP and BIS was greater than or equal to $15 \%$, it was considered to be positive; if the amplitude was less than $15 \%$, it was considered to be negative. According to the principle of sequential allocation method, the test would be terminated when more than or equal to 6 waveforms alternating from a positive direction to a negative direction appeared.

The mean values of the end tidal concentrations of sevoflurane and desflurane were selected at each pair of crossings, which were the concentrations when half of the patients had no response to plantar acupuncture. According to the principle of sequential allocation method, MAC values of sevoflurane and desflurane and $95 \%$ confidence interval (CI) were calculated.

If the MAP value was less than $50 \mathrm{mmHg}$ before the skin incision, $6 \mathrm{mg}$ ephedrine (Approval no.: National Medicine Permission no. H42021159; manufacturing enterprise: Hubei 
Table I. Comparisons of baseline data of four groups of patients.

\begin{tabular}{|c|c|c|c|c|c|c|c|}
\hline \multirow[b]{2}{*}{ Group } & \multirow[b]{2}{*}{$\mathrm{n}$} & \multicolumn{2}{|c|}{ Sex } & \multirow[b]{2}{*}{ Age (years) } & \multirow[b]{2}{*}{ Weight (kg) } & \multirow[b]{2}{*}{ Height $(\mathrm{cm})$} & \multirow[b]{2}{*}{ BMI $\left(\mathrm{kg} / \mathrm{m}^{2}\right)$} \\
\hline & & Male & Female & & & & \\
\hline Group D & 20 & 12 & 8 & $50.21 \pm 8.17$ & $64.45 \pm 11.78$ & $165.52 \pm 6.93$ & $22.45 \pm 2.31$ \\
\hline Group S & 20 & 10 & 10 & $52.32 \pm 7.55$ & $66.12 \pm 13.01$ & $168.37 \pm 6.29$ & $22.39 \pm 2.45$ \\
\hline Group C1 & 20 & 6 & 14 & $51.75 \pm 7.33$ & $63.33 \pm 14.08$ & $167.06 \pm 6.42$ & $22.57 \pm 2.60$ \\
\hline Group C2 & 20 & 12 & 8 & $52.51 \pm 7.11$ & $63.22 \pm 14.14$ & $166.72 \pm 6.31$ & $22.49 \pm 2.98$ \\
\hline$\chi^{2}$ or $t$-value & \multicolumn{3}{|c|}{0.480} & 6.457 & 4.251 & 5.085 & 2.059 \\
\hline P-value & \multicolumn{3}{|c|}{0.187} & 0.207 & 0.235 & 0.591 & 0.436 \\
\hline
\end{tabular}

BMI, body mass index.

Kelun Pharmaceutical Co., Ltd.) was intravenously injected, and if the HR was less than 50 beats/min, $0.5 \mathrm{mg}$ atropine (Approval no.: National Medicine Approval no. H42021159; manufacturing enterprise: Hubei Kelun Pharmaceutical Co., Ltd.) was intravenously injected.

Statistical treatments. Statistical analysis of the data was carried out using SPSS v22.0 software (IBM Corp., Armonk, $\mathrm{NY}$, USA). Measurement data were expressed as mean \pm standard deviation $(\overline{\mathrm{x}} \pm \mathrm{s})$, and the one-way analysis of variance was used to reveal the age, height, weight, preoperative fasting time and preoperative infused fluid volume, and to detect the intergroup differences in HR and MAP values at each time point among various groups. The repeated measures analysis of variance was used to analyze the intragroup differences in $\mathrm{HR}$ and MAP values in each group. The pairwise comparisons were detected using Student-Newman-Keuls-q (SNK-q) test. The logistic regression analysis, Dixon method and mean method were used to determine MAC values of sevoflurane and desflurane, respectively. The changes in MAC values of sevoflurane and desflurane were detected via linear regression analysis and correlation analysis so as to determine the sex distribution ratio. $\mathrm{P}<0.05$ was considered to indicate a statistically significant difference.

\section{Results}

Comparisons of general data of four groups of patients. 80 patients completed the test, and intraoperative and postoperative accidents did not occur in the follow-ups. The general conditions of four groups of patients were generally similar $(\mathrm{P}>0.05)$, and there were no significant differences in all indicators during the operation (Table I).

Changes in MAP values of four groups of patients at different time points before anesthesia induction and before and after skin incision. There was no significant difference in the comparison of MAP value at T0 among four groups of patients $(\mathrm{P}>0.05)$. At each time point from T1 to T6, the MAP values of Group D were significantly lower than those of Group $\mathrm{C} 1$, and the differences were statistically different $(\mathrm{P}<0.05)$. The MAP values of Group $\mathrm{S}$ were significantly lower than those of Group C2, and the differences were statistically different $(\mathrm{P}<0.05)$. The differences in MAP values among the four groups were statistically significant $(\mathrm{P}<0.05$; Table II).

Changes in HR values of four groups of patients at different time points before anesthesia induction and before and after skin incision. There was no significant difference in the comparison of $\mathrm{HR}$ value at T0 among four groups of patients (P>0.05). The HR values of Group D were significantly lower than those of Group C1, and the differences were statistically significant $(\mathrm{P}<0.05)$. The MAP values of Group $\mathrm{S}$ were significantly lower than those of Group $\mathrm{C} 2$, and the differences were statistically different $(\mathrm{P}<0.05)$. The differences in MAP values among the four groups were statistically significant $(\mathrm{P}<0.05$; Table III).

Changes in BIS values of four groups of patients at different time points before anesthesia induction and before and after skin incision. There was no significant difference in the comparison of BIS value at T0 among four groups of patients $(\mathrm{P}>0.05)$. The BIS values of Group $\mathrm{D}$ were significantly lower than those of Group $\mathrm{C} 1$, and the differences were statistically significant $(\mathrm{P}<0.05)$. The BIS values of Group $\mathrm{S}$ were significantly lower than those of Group $\mathrm{C} 2$, and the differences were statistically different $(\mathrm{P}<0.05)$. The differences in MAP values among the four groups were statistically significant $(\mathrm{P}<0.05$; Table IV).

Determination of MAC values of sevoflurane and desflurane by mean method. The MAC values of sevoflurane and desflurane were calculated using the mean method. The $\mathrm{MAC}_{\text {Mean }}$ value was $2.17 \pm 0.13 \%$ (95\% CI: $2.00-2.31 \%$ ) in Group D, $2.09 \pm 0.17 \%(95 \%$ CI: $2.01-2.32 \%)$ in Group S, $3.13 \pm 0.11 \%$ (95\% CI: $3.01-3.35 \%$ ) in Group C1 and 3.15 $\pm 0.12 \%$ (95\% CI: 3.05-3.57\%) in Group C2. Comparisons of $\mathrm{MAC}_{\text {Mean }}$ values of sevoflurane and desflurane among four groups of patients showed that $\mathrm{MAC}_{\text {Mean }}$ values of Group D were significantly lower than those of Group $\mathrm{C} 1$, and the differences were statistically significant $(\mathrm{P}<0.05) ; \mathrm{MAC}_{\text {Mean }}$ values of Group $\mathrm{S}$ were significantly lower than those of Group $\mathrm{C} 2$, and the differences were statistically significant $(\mathrm{P}<0.05)$. The differences in $\mathrm{MAC}_{\text {Mean }}$ values among four groups were statistically significant $(\mathrm{P}<0.05$; Table $\mathrm{V})$. 
Table II. Changes in MAP of four groups of patients at different time points before anesthesia induction and before and after skin incision.

\begin{tabular}{lcccccccc}
\hline Group & $\mathrm{n}$ & $\mathrm{T} 0$ & $\mathrm{~T} 1$ & $\mathrm{~T} 2$ & $\mathrm{~T} 3$ & $\mathrm{~T} 4$ & $\mathrm{~T} 5$ & T6 \\
\hline Group D & 20 & $90.25 \pm 5.13$ & $60.88 \pm 5.22^{\mathrm{a}}$ & $62.50 \pm 5.29^{\mathrm{a}}$ & $63.37 \pm 5.61^{\mathrm{a}}$ & $64.59 \pm 5.87^{\mathrm{a}}$ & $66.32 \pm 6.03^{\mathrm{a}}$ & $67.06 \pm 6.33^{\mathrm{a}}$ \\
Group S & 20 & $91.33 \pm 5.59$ & $62.02 \pm 5.31^{\mathrm{b}}$ & $63.15 \pm 5.12^{\mathrm{b}}$ & $64.37 \pm 5.50^{\mathrm{b}}$ & $65.45 \pm 5.99^{\mathrm{b}}$ & $67.09 \pm 5.25^{\mathrm{b}}$ & $68.18 \pm 5.27^{\mathrm{b}}$ \\
Group C1 & 20 & $88.71 \pm 5.01$ & $66.21 \pm 6.01$ & $67.30 \pm 6.26$ & $67.54 \pm 6.54$ & $70.07 \pm 6.72$ & $73.92 \pm 6.88$ & $76.39 \pm 6.99$ \\
Group C2 & 20 & $88.92 \pm 5.34$ & $67.56 \pm 6.21$ & $69.59 \pm 6.77$ & $70.32 \pm 6.79$ & $72.55 \pm 6.98$ & $76.90 \pm 7.21$ & $77.99 \pm 7.45$ \\
F-value & & 1.083 & 6.371 & 6.593 & 5.427 & 6.486 & 8.443 & 14.477 \\
P-value & & 0.362 & 0.0007 & 0.0005 & 0.0011 & 0.0006 & 0.0004 & 0.0000 \\
\hline
\end{tabular}

aP $<0.05$ Group D vs. Group C1; ${ }^{\mathrm{b}}<0.05$ Group S vs. Group C2. MAP, minimum alveolar concentration.

Table III. Changes in HR of four groups of patients at different time points before anesthesia induction and before and after skin incision.

\begin{tabular}{|c|c|c|c|c|c|c|c|c|}
\hline Group & $\mathrm{n}$ & T0 & $\mathrm{T} 1$ & $\mathrm{~T} 2$ & $\mathrm{~T} 3$ & $\mathrm{~T} 4$ & T5 & T6 \\
\hline Group D & 20 & $87.52 \pm 5.96$ & $73.07 \pm 6.88^{a}$ & $70.22 \pm 6.53^{a}$ & $68.19 \pm 6.73^{a}$ & $75.72 \pm 6.90^{\mathrm{a}}$ & $78.14 \pm 7.52^{\mathrm{a}}$ & $78.99 \pm 7.83^{\mathrm{a}}$ \\
\hline Group S & 20 & $89.59 \pm 6.10$ & $74.01 \pm 6.21^{\mathrm{b}}$ & $71.05 \pm 6.31^{\mathrm{b}}$ & $69.97 \pm 6.81^{\mathrm{b}}$ & $74.99 \pm 6.93^{b}$ & $79.83 \pm 6.99^{b}$ & $80.07 \pm 7.92^{b}$ \\
\hline Group C1 & 20 & $85.23 \pm 5.66$ & $78.15 \pm 7.03$ & $75.32 \pm 7.01$ & $73.81 \pm 6.82$ & $78.15 \pm 7.03$ & $81.33 \pm 7.95$ & $88.45 \pm 8.52$ \\
\hline Group C2 & 20 & $85.91 \pm 5.83$ & $79.31 \pm 7.42$ & $75.07 \pm 6.98$ & $72.91 \pm 6.52$ & $80.56 \pm 7.69$ & $85.68 \pm 7.92$ & $90.39 \pm 9.21$ \\
\hline$F$-value & & 2.622 & 3.927 & 3.128 & 2.978 & 3.342 & 4.127 & 4.595 \\
\hline P-value & & 0.0567 & 0.0116 & 0.0305 & 0.0365 & 0.0213 & 0.0186 & 0.0133 \\
\hline
\end{tabular}

${ }^{\mathrm{a}} \mathrm{P}<0.05$ Group D vs. Group C1; $\mathrm{b}<0.05$ Group S vs. Group C2. HR, heart rate.

Table IV. Changes in bispectral index values of four groups of patients at different time points before anesthesia induction and before and after skin incision.

\begin{tabular}{|c|c|c|c|c|c|c|c|c|}
\hline Group & $\mathrm{n}$ & T0 & $\mathrm{T} 1$ & $\mathrm{~T} 2$ & $\mathrm{~T} 3$ & $\mathrm{~T} 4$ & T5 & T6 \\
\hline Group D & 20 & $93.23 \pm 6.90$ & $81.21 \pm 6.01^{\mathrm{a}}$ & $67.30 \pm 6.26^{\mathrm{a}}$ & $61.54 \pm 6.54^{\mathrm{a}}$ & $54.07 \pm 6.72^{\mathrm{a}}$ & $48.92 \pm 6.88^{a}$ & $46.39 \pm 6.99^{a}$ \\
\hline Group S & 20 & $91.56 \pm 6.21$ & $80.56 \pm 6.21^{b}$ & $66.59 \pm 6.77^{b}$ & $60.32 \pm 6.79^{b}$ & $52.55 \pm 6.98^{b}$ & $46.90 \pm 6.21^{\mathrm{b}}$ & $47.99 \pm 7.45^{\mathrm{b}}$ \\
\hline Group C1 & 20 & $93.52 \pm 6.77$ & $85.08 \pm 5.22$ & $72.50 \pm 5.29$ & $66.37 \pm 5.61$ & $58.59 \pm 5.87$ & $53.32 \pm 6.03$ & $57.06 \pm 6.33$ \\
\hline Group C2 & 20 & $93.84 \pm 6.82$ & $87.34 \pm 5.31$ & $73.15 \pm 5.12$ & $67.37 \pm 5.50$ & $57.45 \pm 5.99$ & $55.09 \pm 5.25$ & $58.18 \pm 5.27$ \\
\hline F-value & & 0.46 & 3.927 & 3.247 & 4.957 & 3.683 & 4.325 & 4.568 \\
\hline P-value & & 0.709 & 0.0129 & 0.0322 & 0.0065 & 0.0232 & 0.0166 & 0.0147 \\
\hline
\end{tabular}

${ }^{\mathrm{a}} \mathrm{P}<0.05$ Group D vs. Group C1; ${ }^{\mathrm{b}}<0.05$ Group S vs. Group C2.

Determination of MAC values of sevoflurane and desflurane by Dixon method. The MAC values of sevoflurane and desflurane were calculated using the Dixon method. The $\mathrm{MAC}_{\text {Dixon }}$ value was $2.18 \%$ (95\% CI: $2.11-2.42 \%$ ) in Group D, $2.03 \%$ (95\% CI: 2.01-2.53\%) in Group S, 3.08\% (95\% CI: 3.04-3.52\%) in Group C1 and 3.109\% (95\% CI: 3.04-3.59\%) in Group C2. Comparisons of $\mathrm{MAC}_{\text {Dixon }}$ values of sevoflurane and desflurane among four groups of patients showed that $\mathrm{MAC}_{\text {Dixon }}$ values of Group D were significantly lower than those of Group C1, and the differences were statistically significant $(\mathrm{P}<0.05) ; \mathrm{MAC}_{\text {Dixon }}$ values of Group $S$ were significantly lower than those of Group $\mathrm{C} 2$, and the differences were statistically significant $(\mathrm{P}<0.05)$.
The differences in $\mathrm{MAC}_{\mathrm{Dixon}}$ values among four groups were statistically significant $(\mathrm{P}<0.05$; Table VI).

Logistic regression analyses of MAC values of sevoflurane and desflurane. Logistic regression analyses showed that the MAC values of sevoflurane and desflurane were closely related to whether patients received neoadjuvant chemotherapy, and $\mathrm{MAC}_{\mathrm{Log}}$ values of sevoflurane and desflurane were decreased in patients receiving neoadjuvant chemotherapy. The $\mathrm{MAC}_{\mathrm{Log}}$ value was $2.19 \%$ (95\% CI: $2.10-2.51 \%$ ) in Group D, $2.05 \%$ (95\% CI: $2.00-2.47 \%$ ) in Group S, 3.08\% (95\% CI: $3.03-3.55 \%$ ) in group C1 and 3.09\% (95\% CI: 3.04-3.59\%) in Group C2. The 
Table V. Determination of MAC values of sevoflurane and desflurane by mean method.

\begin{tabular}{|c|c|c|}
\hline Group & $\mathrm{n}$ & $\mathrm{MAC}_{\text {Mean }}(\%)$ \\
\hline Group D & 20 & $2.17 \pm 0.13^{\mathrm{a}}$ \\
\hline Group S & 20 & $2.09 \pm 0.17^{\mathrm{b}}$ \\
\hline Group C1 & 20 & $3.13 \pm 0.11$ \\
\hline Group C2 & 20 & $3.15 \pm 0.12$ \\
\hline$F$-value & \multicolumn{2}{|c|}{377.547} \\
\hline P-value & \multicolumn{2}{|c|}{0.000} \\
\hline
\end{tabular}

${ }^{\mathrm{a}} \mathrm{P}<0.05$ Group D vs. Group $\mathrm{C} 1$; ${ }^{\mathrm{b}} \mathrm{P}<0.05$ Group $\mathrm{S}$ vs. Group $\mathrm{C} 2$. $\mathrm{MAC}$, minimum alveolar concentration.

Table VI. Determination of MAC values of sevoflurane and desflurane by the Dixon method $(\mathrm{n}=20)$.

\begin{tabular}{|c|c|c|}
\hline Group & $\mathrm{n}$ & $\operatorname{MAC}_{\text {Dixon }}(\%)$ \\
\hline Group D & 20 & 2.18 \\
\hline Group S & 20 & 2.03 \\
\hline Group C1 & 20 & 3.08 \\
\hline Group C2 & 20 & 3.09 \\
\hline$F$-value & \multicolumn{2}{|c|}{118.322} \\
\hline P-value & \multicolumn{2}{|c|}{0.000} \\
\hline
\end{tabular}

MAC, minimum alveolar concentration.

differences in $\mathrm{MAC}_{\mathrm{Log}}$ values among four groups of patients were significant $(\mathrm{P}<0.05)$, and the comparisons between Group D and Group C1 and between Group S and Group C2 showed $\mathrm{P}<0.05$ (Table VII).

Variation trend of sevoflurane and desflurane concentrations to stimulus responses of skin incision. According to the principle of sequential allocation method, the variation trend graphs of different end tidal concentrations of sevoflurane and desflurane to stimulus responses in four groups of patients were obtained (Figs. 1-4).

\section{Discussion}

HCC complicated with jaundice rarely occurs, and lesions often occur in the late tumor phase with a relatively lower surgical resection rate and a relatively higher incidence rate of complications. Neoadjuvant chemotherapy is a systemic chemotherapy method prior to operation or radiotherapy based on the local treatment of malignant tumors $(14,15)$. At present, the study results of many researchers have gradually confirmed the effect of neoadjuvant chemotherapy in the treatment of malignant tumors, which is significant in patients with malignant tumors, so in practical clinical applications, neoadjuvant chemotherapy has been gradually applied in patients with malignant tumors prior to operation or radiotherapy. However, there is a need for further
Table VII. Determination of MAC values of sevoflurane and desflurane by logistic regression analyses.

\begin{tabular}{lcc}
\hline Group & $\mathrm{n}$ & $\mathrm{MAC}_{\mathrm{Log}}(\%)$ \\
\hline Group D & 20 & 2.19 \\
Group S & 20 & 2.05 \\
Group C1 & 20 & 3.08 \\
Group C2 & 20 & 3.09 \\
$F$-value & & 19.564 \\
P-value & & 0.003
\end{tabular}

MAC, minimum alveolar concentration.

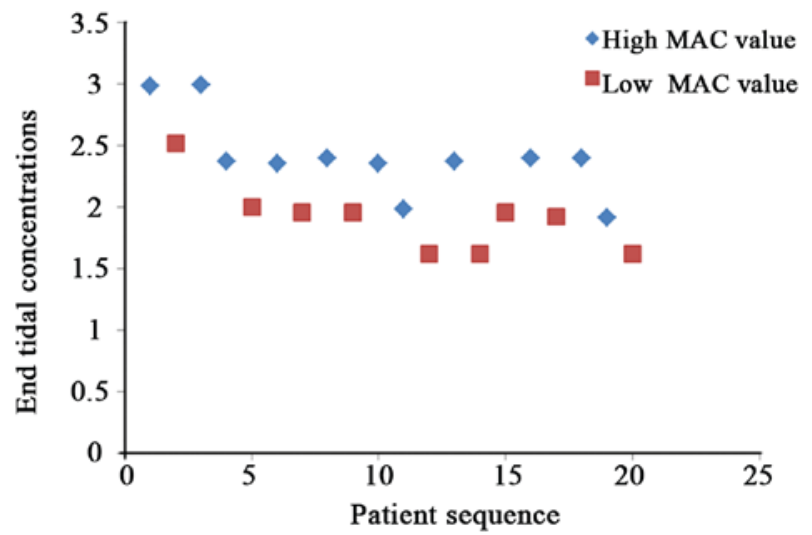

Figure 1. Variation trend graph of different end tidal concentrations of desflurane to stimulus responses of skin incision in patients of Group D Among patients receiving neoadjuvant chemotherapy drugs, the inhibitory effect of patients with high MAC value of desflurane on stimulus responses of skin incision is better than those with low MAC value of desflurane.

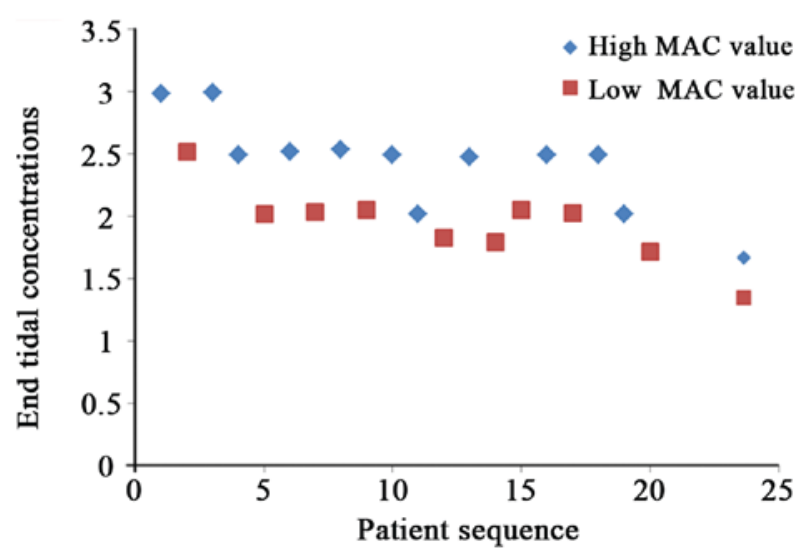

Figure 2. Variation trend graph of different end tidal concentrations of sevoflurane to stimulus responses of skin incision in patients of Group S. Among patients receiving neoadjuvant chemotherapy drugs, the inhibitory effect of patients with high MAC value of sevoflurane on stimulus responses of skin incision is better than those with low MAC value of sevoflurane.

studies on the relationship between chemotherapy drugs and anesthetics, so in this study, a reasonable treatment regimen was selected before operation or radiotherapy. Besides, HCC patients complicated with jaundice with same 


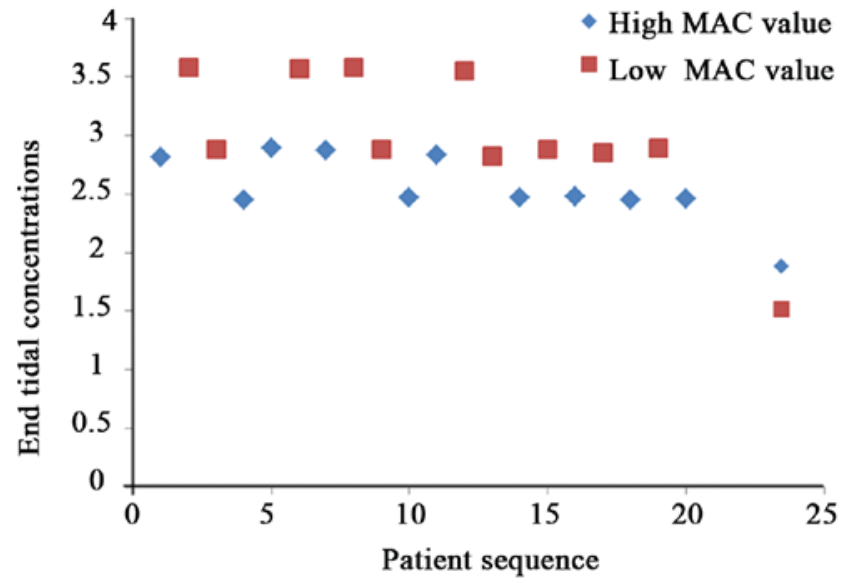

Figure 3. Variation trend graph of different end tidal concentrations of desflurane to stimulus responses of skin incision in patients of Group C1. Among patients receiving neoadjuvant chemotherapy drugs, the inhibitory effect of patients with low MAC value of desflurane on stimulus responses of skin incision is better than those with high MAC value of desflurane.

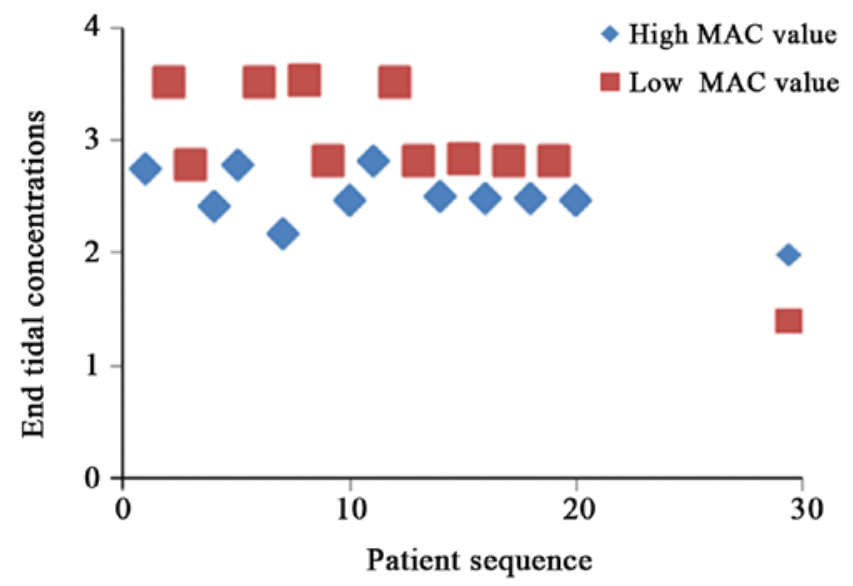

Figure 4. Variation trend graph of different end tidal concentrations of sevoflurane to stimulus responses of skin incision in patients of Group C2. It indicates that among patients receiving neoadjuvant chemotherapy drugs, the inhibitory effect of patients with low MAC value of sevoflurane on stimulus responses of skin incision is better than those with high MAC value of sevoflurane.

treatment courses inhaled sevoflurane and desflurane for anesthesia together with patients receiving no neoadjuvant chemotherapy. MAC values were detected so as to explore whether neoadjuvant chemotherapy could improve the sensitivity of HCC patients complicated with jaundice to sevoflurane and desflurane.

There were no significant differences in the age, sex, height, BMI and other conditions among the three groups of patients. The surgical treatment regimen was to perform preoperative chemotherapy for patients in the neoadjuvant chemotherapy group. The specific action dose of oxaliplatin was $150 \mathrm{mg} / \mathrm{qd}$ and that of tegafur was $60 \mathrm{mg} / \mathrm{bid}$. After the chemotherapy, the computed tomography (CT) was used to assess the tumor changes, and patients were further examined. Operation patients underwent the surgical treatment at the end of 3-week 2 cycles of chemotherapy, thus ensuring the homogeneity within the chemotherapy group.
The test results also revealed that compared with those of patients in Group C, MAC values of patients in Group $\mathrm{S}$ and Group D were reduced, confirming that neoadjuvant chemotherapy could reduce MAC values of HCC patients complicated with jaundice. Chemotherapy leads to the emergence of many adverse reactions in patients, and as the basic principle of selecting neoadjuvant chemotherapy drugs and regimens is the high efficiency and low toxicity, the regimen selected for the neoadjuvant chemotherapy group was oxaliplatin combined with tegafur $(16,17)$. Most of inhaled anesthetics are discharged from the body through the lung, and a small part of them can be discharged from the human body through the skin and urine, but they are not discharged through the liver, so despite of the impact of chemotherapy drugs, the liver function of patients receiving neoadjuvant chemotherapy may be damaged to a certain degree. However, in the body's metabolic process, significant changes will not occur in sevoflurane and desflurane, so that the potency of sevoflurane and desflurane will not be affected. The reason for the decrease in MAC values of halothane and desflurane is likely to the changes in the efficacy of inhaled anesthetics. As the inhalation of anesthetics calms patients down and hypnotize them, it hinders the normal brain function of patients (18-20). Therefore, changes in the brain functional status of patients after neoadjuvant chemotherapy result in decreased MAC values of sevoflurane and desflurane titers (21-23). The MAC value of sevoflurane of patients receiving no chemotherapy detected in the test was $3.08 \%$, which was consistent with that in other studies $(24,25)$.

In summary, preoperative neoadjuvant chemotherapy can reduce MAC values of sevoflurane and desflurane of HCC patients complicated with jaundice. Therefore, from the perspective of improving the safety of anesthesia for patients, the dose of anesthetics needs to be appropriately reduced for patients receiving neoadjuvant chemotherapy. Neoadjuvant chemotherapy can increase the sensitivity of patients to sevoflurane and desflurane, but the specific mechanism of action still needs to be further studied.

\section{Acknowledgements}

Not applicable.

\section{Funding}

No funding was received.

\section{Availability of data and materials}

The datasets used and/or analyzed during the present study are available from the corresponding author on reasonable request.

\section{Authors' contributions}

LZ drafted this manuscript. LZ and XM were responsible for the conception and design of the study. MZ collected the patient data and revised the manuscript critically for important intellectual content. MZ and YD analyzed and interpreted the data. All authors read and approved the final manuscript. 


\section{Ethics approval and consent to participate}

The study was approved by the Ethics Committee of Xiangyang No. 1 People's Hospital, Hubei University of Medicine. Signed written informed consents were obtained from the patients and/or guardians.

\section{Consent for publication}

Not applicable.

\section{Competing interests}

The authors declare that they have no competing interests.

\section{References}

1. Ronellenfitsch U, Schwarzbach M, Hofheinz R, Kienle P, Nowak K, Kieser M, Slanger TE, Burmeister B, Kelsen D, Niedzwiecki D, et al: Predictors of overall and recurrence-free survival after neoadjuvant chemotherapy for gastroesophageal adenocarcinoma: Pooled analysis of individual patient data (IPD) from randomized controlled trials (RCTs). Eur J Surg Oncol 43: 1550-1558, 2017.

2. Kümmel S, Paepke S, Huober J, Schem C, Untch M, Blohmer JU, Eiermann W, Gerber B, Hanusch C, Hilfrich J, et al: Randomised, open-label, phase II study comparing the efficacy and the safety of cabazitaxel versus weekly paclitaxel given as neoadjuvant treatment in patients with operable triple-negative or luminal B/HER2-negative breast cancer (GENEVIEVE). Eur J Cancer 84: 1-8, 2017.

3. Feng X, Chen A, Zhang Y, Wang J, Shao L and Wei L: Central nervous system toxicity of metallic nanoparticles. Int J Nanomedicine 10: 4321-4340, 2015.

4. Winocur G, Henkelman M, Wojtowicz JM, Zhang H, Binns MA and Tannock IF: The effects of chemotherapy on cognitive function in a mouse model: A prospective study. Clin Cancer Res 18: 3112-3121, 2012.

5. Sipocz I, Pinter T, Skaliczky Z and Kullmann T: Effective systemic palliative chemotherapy for intracranial metastases of breast cancer. Orv Hetil 157: 1809-1813, 2016 (In Hungarian).

6. Nudelman KN, McDonald BC, Wang Y, Smith DJ, West JD, O'Neill DP, Zanville NR, Champion VL, Schneider BP and Saykin AJ: Cerebral perfusion and gray matter changes associated with chemotherapy-induced peripheral neuropathy. J Clin Oncol 34: 677-683, 2016.

7. He ZJ, Hu YH and Fan ZY: Median effective effect-site concentration of intravenous anesthetics for loss of consciousness in neoadjuvant chemotherapy patients. Chin Med J (Engl) 124: 504-508, 2011.

8. Trenerry C, Peters MDJ, Corsini N, Damarell RA, Wilson C and Flight I: Patient-reported outcomes following neoadjuvant chemotherapy or chemoradiotherapy treatment for esophageal cancer: A scoping review protocol. JBI Database System Rey Implement Rep 15: 1499-1507, 2017.

9. Weng Y, Yang L, Corringer PJ and Sonner JM: Anesthetic sensitivity of the Gloeobacter violaceus proton-gated ion channel. Anesth Analg 110: 59-63, 2010.

10. Liang Q, Anderson WD, Jones ST, Souza CS, Hosoume JM, Treptow W and Covarrubias M: Positive allosteric modulation of $\mathrm{Kv}$ channels by sevoflurane: Insights into the structural basis of inhaled anesthetic action. PLoS One 10: e0143363, 2015.
11. Klock PA Jr, Czeslick EG, Klafta JM, Ovassapian A and Moss J: The effect of sevoflurane and desflurane on upper airway reactivity. Anesthesiology 94: 963-967, 2001.

12. Kotwani MB and Malde AD: Comparison of maintenance, emergence and recovery characteristics of sevoflurane and desflurane in pediatric ambulatory surgery. J Anaesthesiol Clin Pharmacol 33: 503-508, 2017.

13. Costi D, Cyna AM, Ahmed S, Stephens K, Strickland P, Ellwood J, Larsson JN, Chooi C, Burgoyne LL and Middleton P: Effects of sevoflurane versus other general anaesthesia on emergence agitation in children. Cochrane Database Syst Rev 12: CD007084, 2014

14. Chen VE, Gillespie EF, Zakeri K, Murphy JD, Yashar CM, Lu S and Einck JP: Pathologic response after neoadjuvant chemotherapy predicts locoregional control in patients with triple negative breast cancer. Adv Radiat Oncol 2: 105-109, 2017.

15. Mirkin KA, Luke FE, Gangi A, Pimiento JM, Jeong D, Hollenbeak CS and Wong J: Sarcopenia related to neoadjuvant chemotherapy and perioperative outcomes in resected gastric cancer: A multi-institutional analysis. J Gastrointest Oncol 8: 589-595, 2017.

16. Hentic O, Safi D, Jerome C, Magaly Z, Vinciane R, Sebastien G, Frederique M, Anne C, Philippe L, Philippe R, et al: Neoadjuvant gemcitabine-oxaliplatin (GemOx) combination followed by chemoradiotherapy (CRT) in borderline pancreatic adenocarcinoma (BPC): A promising management. Pancreatology 15 (Suppl): S112, 2015.

17. Seigers R, Loos M, Van Tellingen O, Boogerd W, Smit AB and Schagen SB: Cognitive impact of cytotoxic agents in mice. Psychopharmacology (Berl) 232: 17-37, 2015.

18. Zhou C, Liang P, Liu J, Ke B, Wang X, Li F, Li T, Bayliss DA and Chen X: HCN1 channels contribute to the effects of amnesia and hypnosis but not immobility of volatile anesthetics. Anesth Analg 121: 661-666, 2015.

19. Sun X, Liu J, Wang Y, Bai X, Chen Y, Qian J, Zhu H, Liu F, Qiu X, Sun S, et al: Methotrexate-cytarabine-dexamethasone combination chemotherapy with or without rituximab in patients with primary central nervous system lymphoma. Oncotarget 8: 49156-49164, 2017.

20. Lutterbeck CA, Kern DI, Machado ÊL and Kümmerer K: Evaluation of the toxic effects of four anti-cancer drugs in plant bioassays and its potency for screening in the context of waste water reuse for irrigation. Chemosphere 135: 403-410, 2015.

21. Liu X, Dingley J, Elstad M, Scull-Brown E, Steen PA and Thoresen M: Minimum alveolar concentration (MAC) for sevoflurane and xenon at normothermia and hypothermia in newborn pigs. Acta Anaesthesiol Scand 57: 646-653, 2013.

22. Mishra RK, Mahajan C, Prabhakar H, Kapoor I and Bithal PK: Effect of nitrous oxide on bispectral index values at equi-minimum alveolar concentrations of sevoflurane and desflurane. Indian J Anaesth 61: 482-485, 2017.

23. Du W, Li C, Wang H, Zhao A, Shen J, Yong F and Jia H: Effect of neoadjuvant chemotherapy on sevoflurane MAC-BAR value of patients undergoing radical stomach carcinoma surgery. Int J Clin Exp Med 8: 5649-5657, 2015.

24. Voulgaris DA, Egger CM, Seddighi MR, Rohrbach BW, Love LC and Doherty TJ: The effect of nitrous oxide on the minimum alveolar concentration (MAC) and MAC derivatives of isoflurane in dogs. Can J Vet Res 77: 131-135, 2013.

25. Suarez MA, Seddighi R, Egger CM, Rohrbach BW, Cox SK, KuKanich BK and Doherty TJ: Effect of fentanyl and lidocaine on the end-tidal sevoflurane concentration preventing motor movement in dogs. Am J Vet Res 78: 12-16, 2017.

This work is licensed under a Creative Commons Attribution-NonCommercial-NoDerivatives 4.0 International (CC BY-NC-ND 4.0) License. 